\title{
Antiandrogen Therapy
}

National Cancer Institute

\section{Source}

National Cancer Institute. Antiandrogen Therapy. NCI Thesaurus. Code C15481.

A therapeutic regimen that utilizes pharmaceuticals to reduce serum levels of male sex hormones. 